\title{
PURIFICATION OF RECOMBINANT HUMAN INTERLEUKIN-3 EXPRESSED AS INCLUSION BODIES IN Escherichi coli
}

\author{
Nguyen Thi Quy ${ }^{1}$, Dao Trong Khoa ${ }^{1}$, Duong Thu Huong ${ }^{1}$, \\ Le Thi Thu Hong ${ }^{1}$, Truong Nam Hai ${ }^{1,2, *}$ \\ ${ }^{1}$ Institute of Biotechnology, VAST, Vietnam \\ ${ }^{2}$ Graduate University of Science and Technology, VAST, Vietnam
}

Received 19 July 2019, accepted 12 March 2021

\begin{abstract}
Human interleukin-3 (IL-3) is a hematopoietic growth factor involved in the survival, proliferation and differentiation of multipotent hematopoietic cells. However, recombinant IL-3 is usually expressed as insoluble form (inclusion bodies) in Escherichia coli cells. This state of protein often shows no bioactivity. Herein, we report a simple method for solubilization, refolding and purification of recombinant human IL-3 expressed in E. coli cells. First, IL-3 was expressed in E. coli JM109 (DE3) after being induced with $0.05 \mathrm{mM}$ IPTG at $25^{\circ} \mathrm{C}$. Under these conditions, IL-3 was produced as inclusion bodies with molecular weight of approximately 15 $\mathrm{kDa}$ on SDS-PAGE gel (14\%). Next, IL-3 pellet was separated from the host soluble proteins using sonication followed centrifugation. Then, two strong denaturants such as urea or guanidine hydrochloride were used to test solubilization of the insoluble IL-3. After that, the resulting soluble IL-3 was renatured and subjected to gel filtration chromatography to collect purified IL-3 protein. Our results showed that fractionates contained a single band of IL-3 with recovery rate of about $30 \%$. Several characteristics of recombinant IL-3 were then analyzed. The cytokine IL-3 showed its high purity with a sharp peak on RP-HPLC chromatagram. The Western blot showed a clear signal band on PVDF membrane to demonstrate its right antigenecity against human IL-3 antibody. Besides, amino acid sequence of this cytokine was confirmed by mass spectrophotometry method. The purified IL-3 cytokine is a potential material for further tests.
\end{abstract}

Keywords: Escherichia coli JM109, amino acid sequence, inclusion body, protein refolding, protein purification, recombinant human IL-3.

Citation: Nguyen Thi Quy, Dao Trong Khoa, Duong Thu Huong, Le Thi Thu Hong, Truong Nam Hai, 2021. Purification of recombinant human interleukin-3 expressed as inclusion bodies in Escherichi coli. Academia Journal of Biology, 43(1): 91-98. https://doi.org/10.15625/2615-9023/13973

*Corresponding author email: tnhai@ibt.ac.vn

(O2021 Vietnam Academy of Science and Technology (VAST) 


\section{INTRODUCTION}

Interleukin-3 (IL-3) is a potent growth promoting cytokine involves in regulating biological responses such as cell proliferation, survival and differentiation (Clark \& Kamen, 1987). The main role of IL-3 is to stimulate and differentiate pre-myeloid cells into erythrocytes, granulocytes, monocytes, megakaryocytes, and mast cells (Dexter \& Spooncer, 1987). The human IL-3 gene encodes a protein which is 152 amino acids long, and the mature human IL-3 protein is a 15-17 kDa glycoprotein containing 133 amino acids with two conserved asparagines for potential N-linked glycosylation. However, a research conducted by Ziltener and his coworkers showed that there was no difference in potency between glycosylated and nonglycosylated IL-3 in treated mice (Ziltener et al., 1994). This facilitates the expression of recombinant IL-3 protein using prokaryotic expression system that does not own a complicated process of post-translation.

In fact, recombinant human IL-3 has been synthesized in several expression systems, including bacteria, streptomyces, yeast, Chinese hamster ovary (CHO) cells and baculovirus (Ding et al., 2003; Yang et al., 1986; Yun et al., 2001). Previously, IL-3 was produced using $E$. coli expression system with a low recovery rate of soluble protein, much lower level expression in $\mathrm{CHO}$ cells and even lower amount in baculovirus insect cell expression system $(0.24 \mathrm{mg} / \mathrm{L})$ (Yang et al., 1986; Yun et al., 2001). Remarkably, the protein needs to be purified appropriately after expression to maintain its biological activity.

In our previous report (Duong Thu Huong et al., 2016), we showed that a human il-3 gene fragment with the signal peptide pelB was inserted in pET22b(+) at NdeI and Not I positions then transformed in E. coli JM109 (DE3) cells. After changing several expression conditions such as temperature, IPTG concentration, culture media etc., IL-3 was expressed and precipitated as inclusion form $(\sim 15 \mathrm{kDa})$ in host cells. The insoluble IL-3 need a suitable purification steps to be converted into soluble state. Here, we show a suitable method for obtaning recombinant human IL-3 from E. coli cells. This soluble protein is a potential material for further tests.

\section{MATERIALS AND METHODS}

E. coli JM109 harboring plasmid pET22_IL-3 as reported in our previous paper (Duong Thu Huong et al., 2016) was used to express recombinant human IL-3. Hiload_16/60/Superdex ${ }^{\mathrm{TM}} 75$ column $(121 \mathrm{ml})$ connected to an FPLC system (Amersham Biosciences, USA) was used to detect solubilized IL-3. Besides, Bio wide pore C5 column $(10 \times 10 \mathrm{~cm})$ connected to an HPLC system (Shimadzu, Japan) was used to analyze the IL-3 purity. To detect IL-3 in the purified sample, anti human IL-3 polyclonal antibody (BS7802, Bioworld) was used in Western blotting technique. Other chemicals of analytical grade were purchased from famous companies such as Sigma, Merck, Fermentas, Biorad, Prolabo, etc.

\section{Expression of recombinant proteins}

The expression of recombinant IL-3 in $E$. coli JM109 was previously reported in (Duong Thu Huong et al., 2016). In brief, a colony was cultured overnight at $37^{\circ} \mathrm{C}$ in $5 \mathrm{ml} \mathrm{LB}$ medium supplemented with $100 \mu \mathrm{g} / \mathrm{ml}$ ampicillin (LBamp). The culture was then inoculated with $200 \mathrm{ml}$ fresh LBamp medium (initial $\mathrm{OD}_{600}$ 0.1 ) and grown to an $\mathrm{OD}_{600}$ of 1.0 at $25{ }^{\circ} \mathrm{C}$, after which $0.05 \mathrm{mM}$ IPTG was added to the broth and growth continued for an additional 6 hrs. Cells were harvested at $5.000 \mathrm{rpm}$ for 5 min at $4{ }^{\circ} \mathrm{C}$. Protein expression level was estimated by SDS-PAGE.

\section{Isolation and solubilization of IL-3 inclusion bodies}

The cell pellet was resuspended in $20 \mathrm{mM}$ Tris buffer (pH 7.4) until $\mathrm{OD}_{600}$ was about 10. Cells were lysed by sonication followed by centrifugation at $13000 \mathrm{rpm}$ for $10 \mathrm{~min}$ at 4 ${ }^{\circ} \mathrm{C}$ to collect inclusion bodies. The pellet was then solubilized in $2.5 \mathrm{ml}$ of $20 \mathrm{mM}$ Tris buffer containing $2 \mathrm{M}$ to $8 \mathrm{M}$ guanidine hydrochloride $(\mathrm{GuHCl})$. The suspensions were left at room temperature with gentle vortexing for half an hour. Next, the samples 
were centrifuged at $13.000 \mathrm{rpm}$ for $10 \mathrm{~min}$ to separate soluble phase from insoluble one. The insoluble tube was filled up to $2.5 \mathrm{ml}$ with same buffer. To check IL-3 solubility, the pellet and supernatant phases were analyzed on SDS-PAGE gel.

\section{Refolding and purification of recombinant human IL-3 protein}

The soluble IL-3 phase containing $\mathrm{GuHCl}$ $6 \mathrm{M}$ was diluted to a final concentration of 2 $\mathrm{M} \mathrm{GuHCl}$ by refolding buffer. Following this step, $\sim 4 \mathrm{ml}$ of this phase $(\sim 4,3 \mathrm{mg}$ protein) was loaded on a gel filtration column Superdex G75 previously equilibrated with 50 $\mathrm{mM}$ sodium phosphate buffer ( $\mathrm{pH}$ 7.4) containing $150 \mathrm{mM} \mathrm{NaCl}$ and connected to an FPLC system (Amersham Biosciences). Proteins were detected at $280 \mathrm{~nm}$ with an isocratic rate of $2 \mathrm{ml} / \mathrm{min}$. The $1-\mathrm{ml}$ fractions were collected at a peak of $45^{\text {th }} \mathrm{min}$. The fractionates showing a single band corresponding to IL-3 on SDS-PAGE gel were pooled together and lyophylized. The protein was further analyzed for its purity, antigenecity and amino acid sequence.

\section{Purity and antigenecity analysis of purified IL-3 protein}

Purity of IL-3 was analyzed by reverse phase HPLC and SDS-PAGE. Lyophylized IL-3 was redissolved in A buffer $(0.5 \%$ citric acid, $\left.99.5 \% \mathrm{H}_{2} \mathrm{O}\right)$ to reach $500 \mu \mathrm{l}(1 \mathrm{mg} / \mathrm{ml}$ protein) then loaded on C5 column connected to an HPLC system. The gradient program was set at $5 \mathrm{ml} / \mathrm{min}$ with $\mathrm{A}$ and $\mathrm{B}$ buffers as follows: First 0-5 min.: 0-20\% buffer B (60\% isopropanol, $0.5 \%$ citric acid, $39.5 \%$ $\mathrm{H}_{2} \mathrm{O}$ ); Next 6-20 min.: $100 \%$ buffer B; Final 20-25 min.: $20 \%$ buffer B. Protein eluted was fractionated and subjected to SDS-PAGE electrophoresis.

Antigenicity of recombinant IL-3 was analyzed by Western blotting technique. About $1 \mu \mathrm{g}$ of purified IL-3 was loaded on SDS-PAGE gel and electroblotted to PVDF membrane. Anti IL-3 polyclonal antibody (BS7802, Bioworld) was used to detect the presence of IL-3.

\section{Determination of amino acid sequence of recombinant IL-3}

The protein area of interest was cut off from SDS-PAGE gel and sent to Mission Biotech (Taiwan) for amino acid sequencing by Mass spectrophotometry (MS/MS) analysis. The protein band was washed to remove Coomassie briliant blue followed by vacuum drying. Protein was then reduced by DTT and alkylated by IAA for an hour. Next, protein was treated with Trypsin to be hydrolyzed into peptides. Peptides was then fractionated via HPLC connected to a mass spectrophotometry system. Mass spectral data of peptides was identified by Mascot v1.8 sorfware (MatrixScience Ltd., London, England) using protein data bank of Homo sapiens as control.

\section{RESULTS AND DISCUSSION}

\section{Isolution and solubilization of IL-3 inclusion bodies from $E$. coli cells}

Production of bioactive proteins in E. coli requires standard conditions for solubilizing and refolding proteins if they accumulate as inclusion bodies. As reported in our previous paper, recombinant human IL-3 was well expressed in E. coli JM109 as inclusion form. Western blotting analysis has confirmed that the expressed protein was recombinant human IL-3 (Duong Thu Huong et al., 2016). This protein requires several purification steps to obtain soluble form for potential bioactivity in further studies. Our research presents a method for solubilizing, refolding and purifying recombinant IL-3.

First, IL-3 pellet will be collected by sonicating and centrifugating $25 \mathrm{ml}$ of $E$. coli cell (OD 10). It is known that all host proteins were expressed as soluble form for cell functions. However recombinant IL-3 was produced as inclusion form due to some unknown reasons. When host cells were lyzed by sonicating, all cell components were librated in buffer (lane T). After centrifugating, the host soluble proteins will be in supernatant phase (lane S) while IL-3 proteins precipated. This step helped to 
separate most of the host soluble proteins from IL-3 pellets. Then IL-3 aggregates were isolated and visiualized on SDS-PAGE gel (Fig. 1a, Lane P).
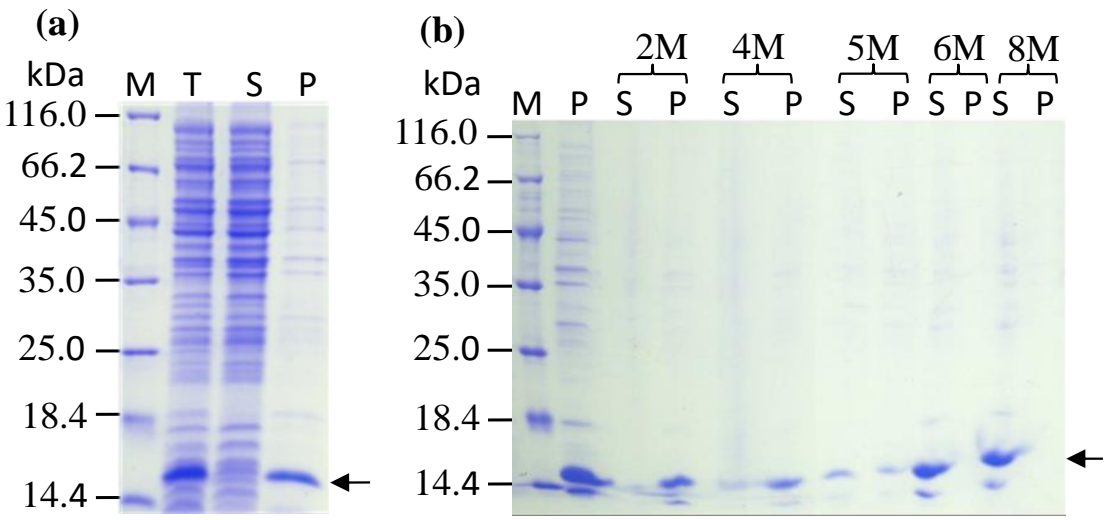

Figure 1. SDS-PAGE analysis of isolated IL-3 inclusion bodies from cell lysate (a); and solubilization of IL-3 pellet in guanidine hydrochloride (b)

Lane M: Protein marker (Fermentas, SM0431); Lane T: Total cell lysate after sonication; Lane S and P: Supernatant and pellet phases obtained after centrifugation, respectively. The collected IL-3 was marked by an arrow at a position of $\sim 15 \mathrm{kDa} ; 2 \mathrm{M}-8 \mathrm{M}$ : Guanindine hydrochloride concentrations in $20 \mathrm{mM}$ Tris $\mathrm{HCl}$ at $\mathrm{pH} 7.4$.

Solubilization of IL-3 inclusion bodies is an important step to achieve maximum protein amount. Among various denaturants, guanidine hydrochloride $(\mathrm{GuHCl})$ and urea were used to solubilize IL-3 aggregates. They both are strong chaotropic denaturants. Besides, guanidine is known to be a very effective denaturing substance in the breakdown of higher structures and nonspecific binding of protein molecules (Singh and Panda, 2005). In this experiment $\mathrm{GuHCl}$ was added to the sample at different concentrations to dissolve IL-3 pellet. Then both soluble and insoluble phases were electrophorized on SDS-PAGE gel. Our result showed that IL-3 pellets dissolved completely between $6 \mathrm{M}$ and $8 \mathrm{M} \mathrm{GuHCl}$ (Lane S, Fig. 1b). This concentration is in the same range as reported previously for the dissolving recombinant human IL-2 (Sengupta et al., 2008).

Besides, solubilization of IL-3 pellet by urea was also tested. The results showed that IL-3 pellet were only partially solubilized even when urea concentration was increased to $8 \mathrm{M}$ (data not shown here). Consequently, this strong denaturant was not used for further studies. Several other detergents such as SDS and $\mathrm{CTAB}$ were not tested in this experiment due to difficulties in removing their critical miceller concentraion, especially for further pharmaceutical applications. For our experiment, $6 \mathrm{M} \mathrm{GuHCl}$ was chosen to dissolve IL-3 pellets.

\section{Refolding IL-3 protein from denaturing state}

Protein refolding is a process of converting them from a non-coiled to a correct folding state. This is a delicate process with no defined protocol for all proteins. Methods commonly used for renaturation of solubilized protein are dilution, dialysis, gel filtration or immobilization on to a solid support. The principle of re-folding is to gradually reduce the denaturant excess and reducing agents to create a proper environment for protein to fold spontaneously into a correct conformation.

For previously solubilized IL-3 state in 6 $\mathrm{M} \mathrm{GuHCl}$, protein refolding was then initiated by diluting this solution to lower $\mathrm{GuHCl}$ concentrations of $0.5 \mathrm{M}, 1 \mathrm{M}, 1.5 \mathrm{M}, 2 \mathrm{M}, 2.5$ $\mathrm{M}$, and $3 \mathrm{M}$. After an overnight incubation at 
room temperature, the solution was then centrifuged to separate the supernatant from insoluble phase. Our result indicated that IL-3 remained in its soluble state at the lowest concentration of $2 \mathrm{M}$ (lane 2, soluble phase, noted as an arrow, Fig. 2). At more diluted $\mathrm{GuHCl}$ concentration $(0.5$ to $1.5 \mathrm{M})$, the proteins were re-precipitated as shown in insoluble phase on SDS-PAGE gel. For proteins having cysteine residues, a redox reagent containing reduced and oxidized glutathione is suggested to include in refolding buffer for proper disulfide bond formation. Best conditions for refolding of disulfide-bonded proteins were reached when the reduced form is present in excess and the buffer $\mathrm{pH}$ is slightly alkaline. These conditions allows disulfide exchange reaction to occur faster until the protein reaches the most stable disulfide configuration, usually the native form (soluble state) (Sengupta et al., 2008). In fact, IL-3 molecule consists of two cystein residues at $\mathrm{Cys}^{16}$ và $\mathrm{Cys}^{84}$. Therefore, a reduced and oxidized glutathione reduction mixture (10:1) was included in the buffer then denaturant $\mathrm{GuHCl}$ was diluted to a lower concentration (2 $\mathrm{M})$. While contaminated proteins were also removed and denaturing agent was reduced, soluble IL-3 form was maximum. This state facilitates further purification step on a gel filtration system. This $2 \mathrm{M} \mathrm{GuHCl}$ concentration was also reported to dilute IL-2 prior subjecting to gel filtration (Sengupta et al., 2008).

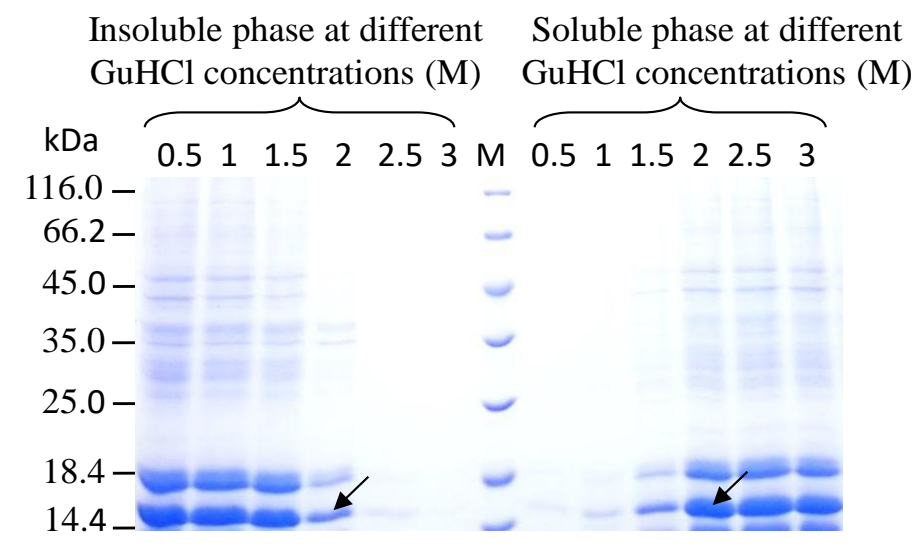

Figure 2. IL-3 proteins were separated into soluble and insoluble phases after diluting $\mathrm{GuHCl}$ from $6 \mathrm{M}$ to lower concentrations Lane M: Protein marker (Fermentas, SM0431)

\section{Purification and characteristic assesment of recombinant IL-3 protein}

The supernatant containing $4.3 \mathrm{mg}$ of protein was loaded on a gel filtration column Superdex 75 connected to an FPLC system. Fig. 3a showed the protein elution profile with 3 main peaks, of which the $45^{\text {th }}$ min. peak (I, Fig. 3a) represented a protein band of $\sim 15$ $\mathrm{kDa}$ corresponding to IL-3 (Lane 1-6, Fig. 3b). While the remaining peaks (II and III, Fig. 3a) indicated no protein bands on gel profile, it seemed that these two peaks represented an eluted certain component $(\mathrm{GuHCl})$ rather than a protein. To verify, the purification process was repeated with $\sim 5.52$ mg protein. In this experiment, chromatographic profile was obtained similarly to the first time. IL-3 protein was eluted at $45^{\text {th }}$ minute and visualized on a silver staining gel (data not shown here). Elution fractions were pooled together and reached to $8 \mathrm{ml}$ corresponding to $1.28 \mathrm{mg}$ protein (Bradford assay). Thus, protein recovery via column was $29.76 \%$ and IL-3 was successfully purified by gel filtration chromatography. As reported previously, recombinant human IL-2 was also purified by gel filtration method and protein yield reached 0,32 g/L (Sengupta et al., 2008). Another 
report conducted by Nguyen et. al., (2018) showed that recombinant human IL-11 was expressed in E. coli cells and purified by affinity chromatography to gain $0.32 \mathrm{~g} / \mathrm{L}$. Protein recovery in the studies was not the

(a)

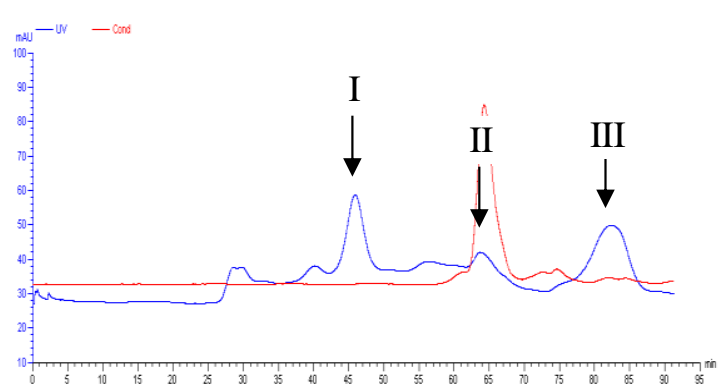

same for each protein type because the purification process largely depended on the nature of the protein, the buffering composition and $\mathrm{pH}$, the chromatographic system, and sample processing, etc. (b)

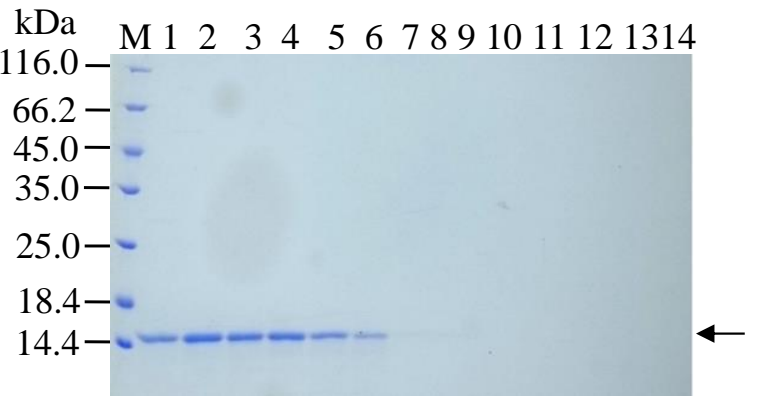

Figure 3. Purification profile of IL-3 by gel filtration chromatography (a) and 14\% SDS-PAGE stained with Coomassie blue R250 (b)

Eution profile of solubilized IL-3 inclusion bodies on Superdex 75 connected to an FPLC system (Amersham Biosciences). The absorbance was measured at $280 \mathrm{~nm}$ and protein was collected in 1-ml fraction size at three peaks marked as I, II and III. Frationates were then electrophoresized on 14\% SDSPAGE gel and visualized by Coomassie staining. Lane M: Protein marker (Fermentas, SM0431); Lane 18; 9-11; and 12-14: protein fractions collected at peak I, II, and III, respectively.

After being purified by gel filtration, IL3 purity was further analyzed by reverse phase chromatography. Only a sharp peak at $10.5^{\text {th }}$ min. appeared on HPLC chromatogram profile (Fig. 4a). Fractionates of 45 to 47 was subjected to SDS-PAGE analysis. Fig. 4b indicated a single band corresponding to a predicted molecular weight of IL-3 (Lane 45-46). Our result also verified that non-interest proteins were removed efficiently through above purification steps. (a)

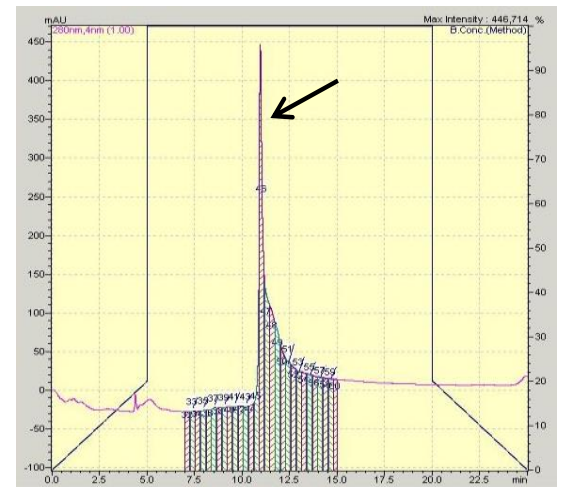

(b)

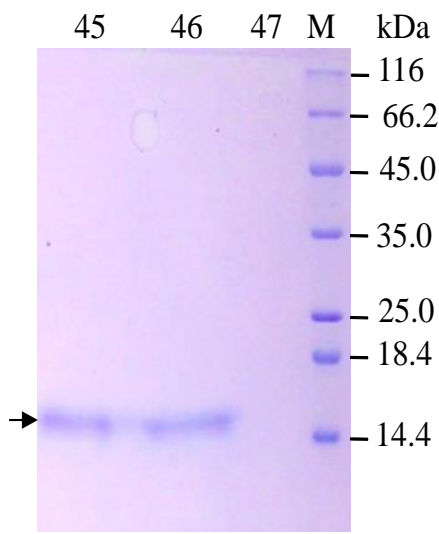

(c)

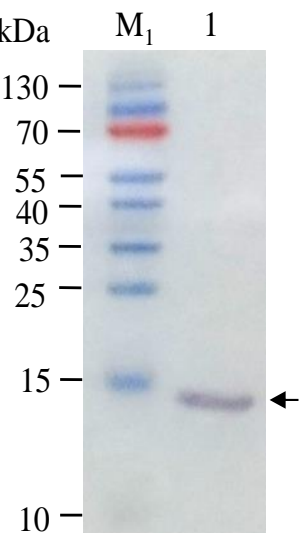

Figure 4. Several analysis of purified IL-3 protein

(a) HPLC chromatogram of purified IL-3 protein. A HPLC system with Biowidepore C5 column (250x10x10) was used to analyze the purity of IL-3. Solvents used to elute IL-3 consists of $39.5 \% \mathrm{H}_{2} \mathrm{O}$, 
$0.5 \%$ citric acid and $60 \%$ isopropanol. The protein was eluted with gradient of $0-100 \%$ B buffer over 20 $\mathrm{min}$. at a flow rate of $5 \mathrm{ml} / \mathrm{min}$ and detected at $280 \mathrm{~nm}$. (b) SDA-PAGE analysis of purified IL-3 eluted at an unique peak on HPLC chmatogram. Lane M: Protein marker (Fermentas, SM0431); Lane 46-47: Fractionates at a peak of $10.5^{\text {th }}$ min. (c) Antigenecity analysis of purified IL-3 using anti IL-3 polyclonal antibody (BS7802, Bioworld). The purified IL-3 was electrophoresized and transblotted on PVDF membrane, followed by hybridization with anti IL-3 antibody. Lane $\mathrm{M}_{1}$ : Pre-stained protein marker (Fermentas, SM0671); Lane 1: Purified IL-3 protein, marked as an arrow.

Antigenic property of a protein refers to its ability to bind to specific antibody. IL-3 existence in the purified sample was further confirmed by Western blotting technique using polyclonal antibody to human IL-3 (Fig. 4c). The only signal band on PVDF membrane was $\sim 15 \mathrm{kDa}$ in length, as predicted by the theoretical molecular weight of human IL-3. The purified sample was therefore recombinant IL-3 protein.

\section{Amino acid sequence of purified IL-3 protein}

The amino acid sequence of the purified IL-3 was sequenced by MS/MS analysis to confirm the peptide's presence, returning a sequence identical to the expected amino acid sequence (Fig. 5). Furthermore, Nterminal amino acid sequencing showed no $\mathrm{Pel \textrm {B }}$ leader. It revealed the correct cleavage of signal peptide during protein processing.

\section{APMTQTTPLK TSWVNCSNMI DEIITHLKQP PLPLLDFNNL NGEDQDILME 51 NNLRRPNLEA FNRAVKSLQN ASAIESILKN LLPCLPLATA APTRHPIHIK 101 DGDWNEFRRK LTFYLKTLEN AQAQQTTLSL AIF}

Figure 5. Amino acids of purified IL-3 protein were sequenced and identified by MS/MS analysis

\section{CONCLUSION}

Recombinant human IL-3 was expressed as inclusion bodies in E. coli JM109 (DE3) with molecular weight of $\sim 15 \mathrm{kD}$ on SDSPAGE gel. Although the proteins were insoluble form, they are purified successfully by denaturing and refolding technique, followed by gel filtration chromatography. IL-3 recovery efficiency via chromatographic step was $29.76 \%$. Analysis of purified protein showed its right antigenicity against human IL-3 polyclonal antibody with the same amino acid sequence as originally designed. Our results indicated that the purified IL-3 can be obtained and used as material for further tests such as endotoxin removal or bioactive analysis on dependent cell lines.

Acknowledgements: This work was funded by the project "Research on production of high quality Interleukin-3 and Interleukin-
11 used in medicine (treatment)" under the management by the Ministry of Science and Technology. Our work used equipment of the Key Laboratory of Genetic Technology at the Institute of Biotechnology, Vietnam Academy of Science and Technology (VAST).

\section{REFERENCES}

Clark S. C., Kamen R., 1987. The human hematopoietic colony-stimulating factors. Science 236: 1229-1237.

Dexter T. M., Spooncer E., 1987. Growth and differentiation in the hemopoietic system. Annu. Rev. Cell Biol., 3: 423-441.

Ding H., Griesel C., Nimtz M., Conradt H. S., Weich H.A., Jäger V., 2003. Molecular cloning, expression, purification, and characterization of soluble full-length, human interleukin-3 with a baculovirusinsect cell expression system. Protein Expr. Purif., 31: 34-41. 
Duong Thu Huong, Nguyen Thi Quy, Dang Thi Ngoc Ha, Le Thi Thu Hong, Truong Nam Hai, 2016. Optimization of fermentation conditions for the expression of Interleukin-3 in fusion with $P e l \mathrm{~B}$ in $E$. coli. Tap chi Sinh hoc, 38(2): 250-256 (in Vietmamese with English summary).

Joseph B. C., Pichaimuthu S., Srimeenakshi S., Murthy M., Selvakumar K., Ganesan M., Manjunath S. R., 2015. An overview of the parameters for recombinant protein expression in Escherichia coli. J Cell Sci Ther., 6: 1-7.

Nguyen T. Q., Duong T. H., Dang T. N. H., Le N. G., Le Q. G., Do T. H., Nguyen V. D., Le T. T. H., Truong N. H., 2018. Enhanced soluble expression and efective purification of recombinant human interleukin-11 by SUMO fusion in Escherichia coli. Indian Journal of Biotechnology, 17: 579-585.

Sengupta P., Meena K., Mukherjee R., Jain S. K., Maithal K., 2008. Optimized conditions for high-level expression and purification of recombinant human interleukin-2 in E. coli. Indian J. Biochem. Biophys., 45: 91-97.

Singh S. M., Panda A. K., 2005. Solubilization and refolding of bacterial inclusion body proteins. J. Biosci. Bioeng., 99: 303-310.

Yang Y. C., Ciarletta A. B., Temple P. A., Chung M. P., Kovacic S., Witek-Giannotti J. S., Leary A. C., Kriz R., Donahue R. E., Wong G. G., 1986. Human IL-3 (multiCSF): identification by expression cloning of a novel hematopoietic growth factor related to murine IL-3. Cell, 47: 3-10.

Yun S. I., Yahya A. R. M., Cossar D., Anderson W. A., Scharer J. M., MooYoung M., 2001. Temperature downshift increases recombinant cytokine titer in Streptomyces lividans fermentation. Biotechnol. Lett., 23: 1903-1905.

Ziltener H. J., Clark-Lewis I., Jones A. T., Dy M., 1994. Carbohydrate does not modulate the in vivo effects of injected interleukin-3. Exp. Hematol., 22: 1070-1075. 